\title{
Increasing Rates of Influenza Vaccination During Pregnancy: A Multisite Interventional Study
}

\author{
David H. Wallis, MD, Jennifer L. Chin, MD, Denise K. C. Sur, MD, and \\ Michael Y. Lee, MD
}

Background: Pregnancy is a high-risk indication for influenza vaccination; however, rates of vaccination fall short of Centers for Disease Control and Prevention-recommended guidelines.

Methods: Brief educational sessions with family physicians and obstetricians were undertaken in the fall of 2002. Notes reading "Think Flu Vaccine" were placed on active obstetric charts during the study period. Charts were reviewed at the end of influenza season for documentation of discussion or administration of influenza vaccination. Charts for the same period during the previous 2 years were also reviewed for baseline.

Results: Baseline rates of vaccination or discussion averaged $1.5 \%$ over the $2000-2002$ influenza seasons. After intervention, the 2002-2003 rate of vaccination or discussion demonstrated an almost 15-fold increase to $21.9 \%$. This was greater in family practices $(3.2 \%$ to $44.9 \%)$ versus obstetric practices $(1.2 \%$ to $19.4 \%)$, and in small $(3.3 \%$ to $46.7 \%)$ versus large $(1.1 \%$ to $16 \%)$ practices (all values were $P<.001)$.

Conclusions: Provider education with simple chart prompts seems an effective way to increase rates of physician discussion of influenza vaccination with pregnant women. The increased rates seen in this study across various practice settings also suggest that inclusion of influenza vaccination on standardized prenatal care flowsheets may achieve similar goals with less individualized effort and should be considered. (J Am Board Fam Med 2006;19:345-9.)

Influenza epidemics are a major health concern in the United States, affecting $10 \%$ to $20 \%$ of the general population and causing significant morbidity and mortality each year. ${ }^{1}$ The influenza vaccine is the best available means to reduce the morbidity and mortality associated with the virus, with a potential $90 \%$ effectiveness in preventing disease in healthy adults under age 65 and up to $60 \%$ in preventing hospitalization. ${ }^{2}$ The Advisory Committee on Immunization Practices (ACIP) of the Centers for Disease Control and Prevention

Submitted 11 September 2005; revised 19 February 2006; accepted 22 February 2006.

From the South Bay Family Medical Group (DHW), Torrance, CA; Peak Health Medical Group (JLC), Los Angeles, CA; Santa Monica-UCLA Family Practice Residency Program (DKCS); David Geffen School of Medicine at University of California (DKCS, MYL), Los Angeles, CA; Clinica Sierra Vista (MYL), Bakersfield, CA.

Funding: Financial support provided by a research stimulation grant from the Joint American Academy of Family Physicians (AAFP)/F-AAFP Grant Awards Council.

Manuscript content was presented in poster format at the AAFP Scientific Assembly in New Orleans, LA, in October 2003.

Conflict of interest: none declared.

Corresponding author: Jennifer L. Chin, MD, Peak Health Medical Group, 2143 S. Sepulveda Boulevard 300, Los Angeles, CA 90025 (E-mail: jchin@peakdocs.com).
(CDC) currently (July 2005) recommends vaccinating high-risk populations who may have an increased morbidity or mortality from complications of influenza (see Table 1), including women who will be pregnant during the influenza season. ${ }^{3}$

Although the vaccine is specifically recommended during pregnancy, many physicians have historically advocated avoidance of vaccination during the first trimester. ${ }^{4}$ This was not due to reported adverse effects, but rather because routinely administering any vaccine during this time may have prompted spurious anecdotal association between vaccination and either spontaneous abortion or birth defects. However, the ACIP guidelines have recently been updated to specifically state that influenza vaccination in any trimester is recommended. ${ }^{3}$ The current recommendation is to provide pregnant women with vaccination in the fall, ideally during the months of October and November, as there seems to be at least a 2 week period to reach peak immunologic response to the vaccine. $^{5}$

Despite the decrease in morbidity and mortality, multiple obstacles may deter influenza vaccination during pregnancy. Previous research has shown 


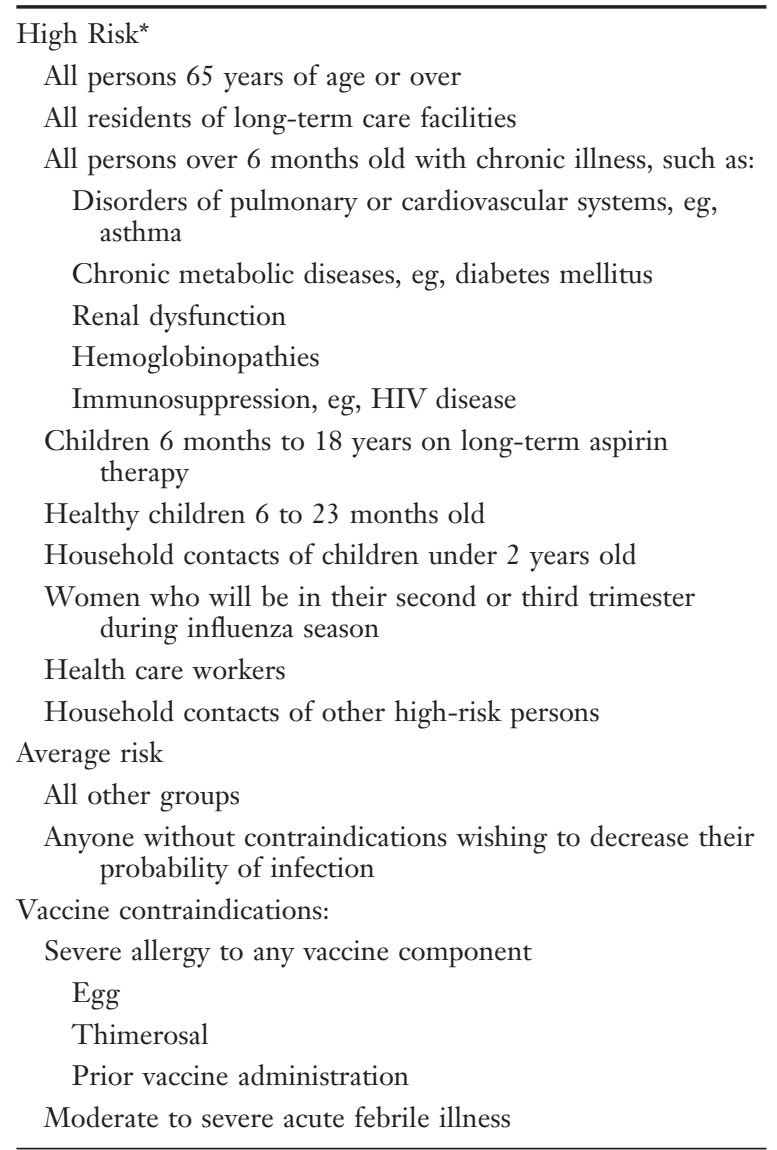

* Current CDC recommendations (2005) advise immunization of all pregnant women, regardless of trimester, as well as conditions such as neuromuscular disorders that can compromise respiratory function or increase the risk for aspiration.

that obstacles may include lack of education of both patients and physicians, oversight by the provider, lack of influenza vaccine supply, and financial cost and physician compensation. ${ }^{6}$ There is a lack of significant research in the efficacy of interventions to improve vaccination rates in these women. One potential intervention is provider prompting during patient visits to increase vaccination rates and reduce missed opportunities. ${ }^{7}$ Determining effective interventions is important in increasing the vaccination rate in this high-risk population.

\section{Materials and Methods}

This prospective, interventional, multisite trial was designed to evaluate the efficacy of chart reminders and physician education as interventions to improve rates of physician discussion of influenza vaccine with pregnant women in a suburban community. Six physician practices with obstetrical privileges at Santa Monica-UCLA Medical Center (Santa Monica, CA) were enrolled in the study. The study population comprised one group obstetric practice, 2 solo private obstetric practices, one group family medicine practice, and 2 small (single provider) family medicine practices. Application was submitted, and study was declared exempt from Institutional Review Board approval. Early in the fall of 2002, each participating physician received a short educational session regarding the indications, contraindications, and background information for the influenza vaccine. Educational sessions were designed to increase knowledge of the most recent ACIP recommendations. These sessions were approximately 5 minutes in length, with a presentation and discussion reviewing the indications, contraindications, and side effect profile of the vaccine with each provider. Reminder notes reading "Think Flu Vaccine" were then placed on the charts of all current obstetric patients, and staff were instructed to do likewise for all new obstetric patients.

Late in the spring of 2003, the charts of obstetric patients with estimated dates of delivery between 1 December 2000 and 31 August 2003 were systematically reviewed. To ensure that a physician-patient relationship had been established and there was opportunity to vaccinate the patient if indicated, patients with fewer than 3 documented visits were excluded. Because the peak of influenza season has historically occurred between the months of December and March, ${ }^{3,4}$ patients for whom influenza vaccine was not indicated (estimated dates of delivery from September 1 through November 30) were also excluded from the study. In addition, patients with no documented visits after 14 weeks' gestation were excluded, due to theoretical concerns surrounding first trimester vaccination that have since been addressed with the most recent ACIP recommendations. ${ }^{3}$

The focus of this study was on improving and monitoring provider compliance with CDC guidelines to advise vaccination in pregnant women. As such, chart review focused on whether discussion of prenatal influenza vaccination was documented by the provider. Documentation of vaccine administration, instruction to obtain vaccine from another physician, or patient refusal of vaccination each demonstrated that the provider remembered to consider vaccination in accordance with $\mathrm{CDC}$ recommendations and thus were grouped together for analysis. 
Table 2. Comparison of Vaccination Discussion Rates, Pre- and Post-Intervention

\begin{tabular}{|c|c|c|c|c|c|c|c|c|c|}
\hline & \multirow[b]{2}{*}{ Total } & \multicolumn{2}{|c|}{ Pre Years:2000-2002 } & \multirow[b]{2}{*}{$\begin{array}{l}\text { Proportion } \\
\text { Pre }(\%)\end{array}$} & \multicolumn{2}{|c|}{ Post Year:2002-2003 } & \multirow[b]{2}{*}{$\begin{array}{c}\text { Proportion } \\
\text { Post (\%) }\end{array}$} & \multirow{2}{*}{$\begin{array}{c}\text { Difference } \\
\text { (Post-Pre) } \\
(\%)\end{array}$} & \multirow[b]{2}{*}{$P$ Value } \\
\hline & & $\begin{array}{c}\mathrm{N} \text { before } \\
\text { Intervention }\end{array}$ & $\begin{array}{l}\text { Discussed } \\
\text { Pre }\end{array}$ & & $\begin{array}{c}\mathrm{N} \text { after } \\
\text { Intervention }\end{array}$ & $\begin{array}{l}\text { Discussed } \\
\text { Post }\end{array}$ & & & \\
\hline Small FP 1 & 29 & 18 & 0 & 0 & 11 & 2 & 18.2 & 18.2 & .136 \\
\hline Small FP 2 & 19 & 8 & 0 & 0 & 11 & 7 & 63.6 & 63.6 & .013 \\
\hline Large FP & 178 & 131 & 5 & 3.8 & 47 & 22 & 46.8 & 43 & $<.001$ \\
\hline Small OB 1 & 99 & 62 & 4 & 6.5 & 37 & 20 & 54.1 & 47.6 & $<.001$ \\
\hline Small OB 2 & 201 & 123 & 3 & 2.4 & 78 & 35 & 44.9 & 42.4 & $<.001$ \\
\hline Large OB & 1558 & 1035 & 8 & 0.8 & 523 & 69 & 13.2 & 12.4 & $<.001$ \\
\hline FP overall & 226 & 157 & 5 & 3.2 & 69 & 31 & 44.9 & 41.7 & $<.001$ \\
\hline OB overall & 1858 & 1220 & 15 & 1.2 & 638 & 124 & 19.4 & 18.2 & $<.001$ \\
\hline Small practice overall & 348 & 211 & 7 & 3.3 & 137 & 64 & 46.7 & 43.4 & $<.001$ \\
\hline Large practice overall & 1736 & 1166 & 13 & 1.1 & 570 & 91 & 16 & 14.8 & $<.001$ \\
\hline Overall & 2084 & 1377 & 20 & 15 & 707 & 155 & 21.9 & 20.5 & $<.001$ \\
\hline
\end{tabular}

Documentation of vaccination advisement from 2000 through 2003 was collected systematically from each practice. These data were then statistically analyzed to determine the efficacy of the educational and reminder intervention described above. All $P$ values were calculated by $\chi^{2}$ statistical analysis, except in the 2 individual family practices where the two-tailed Fisher's exact test was used due to their small sample size.

\section{Results}

A total of 2084 charts met inclusion criteria. Of these, $226(10.8 \%)$ were from family medicine practices and 1858 (89.2\%) were from obstetric practices. Private, solo practices accounted for 348 (16.7\%), whereas $1736(83.3 \%)$ were from group practices.
Baseline rates of influenza vaccination discussion among this study population averaged $1.5 \%$ over the 2000-2001 and the 2001-2002 influenza seasons. After the intervention of physician education and systematic reminders as described above, the 2002-2003 rate of vaccination discussion demonstrated an almost 15 -fold increase to $21.9 \%(P<$ .001; Table 2).

The percentage of patients with whom immunization was discussed and documented rose from $3.2 \%$ to $44.9 \%$ among family practices and from $1.2 \%$ to $19.4 \%$ in obstetric practices (Figure 1). Similarly, immunization discussion rose from $3.3 \%$ to $46.7 \%$ in small practices, and from $1.1 \%$ to $16 \%$ in large practices. Each of these differences was statistically significant with values of $P<.001$.

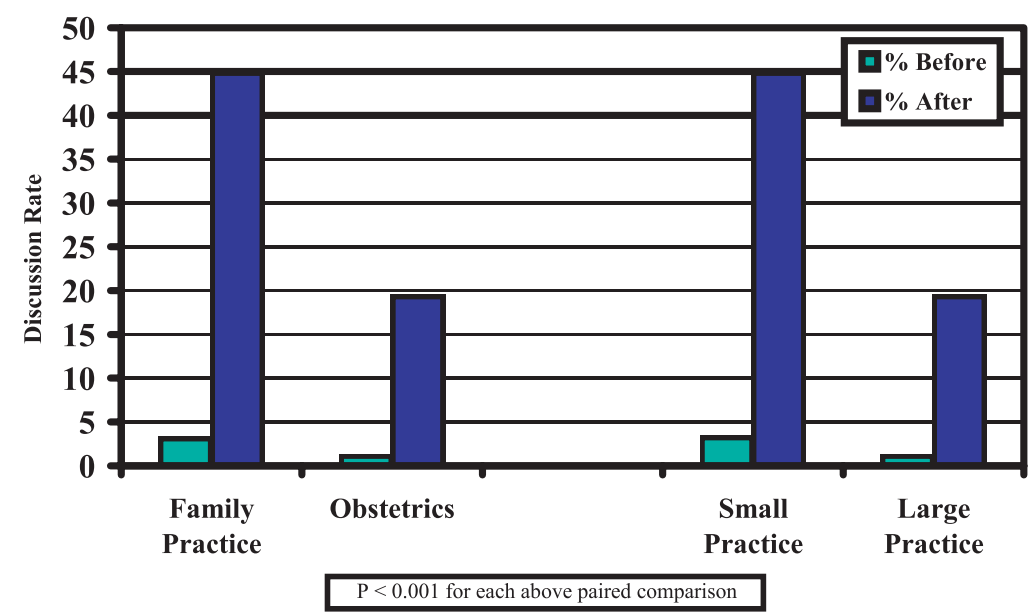

Figure 1. Effects of Intervention by Practice Type and Size. 


\section{Discussion}

Women who are pregnant during the influenza season are classified by the CDC as high risk for developing complications of influenza infection. For health care providers, the primary resource to prevent these complications is the influenza vaccine. Due to many factors, vaccination rates in this high-risk group unfortunately remain extremely low. ${ }^{8-10}$ The importance and safety profile of influenza vaccination during pregnancy has since been further emphasized by the more recent recommendations of the ACIP, ${ }^{3}$ which call for influenza vaccination in all women who will be pregnant during flu season, rather than only those who will be in their second or third trimesters during this time. $^{11}$

Especially in an era of limited financial resources and overwhelming time constraints, interventions that are of low cost and require little labor to implement are intuitively the most desirable. This study demonstrates that education and chart reminders are an effective way to increase patientphysician discussion of the benefits of influenza vaccination among pregnant women. The percentage of women who were advised to be immunized after the intervention rose 15-fold among family physicians and 18-fold among obstetricians to $44.9 \%$ and $19.4 \%$, respectively. Although the fold increase was higher among obstetricians, the actual percentage of increase was greater among family physicians ( $41.7 \%$ vs $18.2 \%$ ). Given that discussion of immunization was documented in nearly half of their patients, compared with less than $1 / 5$ th of obstetricians' patients, the intervention had greater success in family physicians' offices. Similarly, the intervention was more effective in small practices than large ones, as evidenced by a $43.4 \%$ difference versus a $14.8 \%$ increase in large groups. Most importantly, however, these interventions proved to be highly effective in increasing the rates of discussion of influenza vaccination in all studied practice settings: $P$ values for comparisons were highly significant for both family medicine and obstetric practices, as well as in both small individual and large group practices.

Although this intervention did improve rates of vaccination discussion between patient and provider, the overall rates still remained low. Other factors still may play a role in these low immunization discussion rates, and more effective means of physician education than those used in this study may further increase compliance with ACIP recommendations. This study does strongly suggest, however, that significant obstacles in vaccinating this population may include oversight by the provider and/or lack of education regarding the vaccine indications. More importantly, however, it demonstrates that these obstacles are easily surmountable with a simple intervention.

One limitation of this study is the presence of confounding factors that may affect vaccination rates, such as cost or supply of vaccines in the office, marketing, and awareness and desire of the general public regarding the influenza vaccine. $\mathrm{Al}-$ though 2 years of data were recorded from each office to determine the practice's baseline vaccination rates, these variables cannot be held constant and thus may have shifted coincidentally with the intervention being evaluated as no concurrent control group was studied. Errors in documentation may represent another limitation of this study.

Influenza is a common illness with significant morbidity and mortality, for which a widely available, highly effective, and cost-conscious method of prevention has been developed. Pregnancy represents both a time of regularly scheduled interface with the medical community and a time of increased vulnerability to the disease. Optimizing the preventive benefits of these encounters should involve increasing compliance with CDC guidelines for immunization. This study demonstrates that chart reminders are a simple but effective way to increase vaccine discussion rates and require minimal labor and cost. Furthermore, it suggests that inclusion of influenza vaccination status onto standardized prenatal care flowsheets may achieve the same goals with less cost or individualized effort and should, therefore, be considered.

We thank D. Yvette LaCoursiere, MD, MPH, for skill, insight, and assistance in reviewing this manuscript.

\section{References}

1. Dolin R. Infectious disease. In: Braunwald E, et al, editors. Harrison's principles of internal medicine. 15th ed. New York: McGraw-Hill; 2001. p. 112530.

2. Zimmerman RK, Middleton DB, Burns IT, Clover RD. Routine vaccines across the life span, 2003. J Fam Pract 2003;52 (1 Suppl):S1-21. 
3. Centers for Disease Control and Prevention. Prevention and control of influenza: recommendations of the Advisory Committee on Immunization Practices (ACIP). MMWR 2005;54(RR08):1-40.

4. Sur DK, Wallis DH, O'Connell TX. Vaccinations in pregnancy. Am Fam Physician 2003;68:299-304.

5. Gross PA, Russo C, Dran S, Cataruozolo P, Munk G, Lancey SC. Time to earliest peak serum antibody response to influenza vaccine in the elderly. Clin Diagn Lab Immunol 1997;4:491-2.

6. Wallis DH, Chin JL, Sur DK. Influenza vaccination in pregnancy: current practices in a suburban community. J Am Board Fam Pract 2004;17:287-91.

7. Minkovitz CS, Belote AD, Higman SM, Serwint JR, Weiner JP. Effectiveness of a practice-based intervention to increase vaccination rates and reduce missed opportunities. Arch Pediatr Adolesc Med 2001;155:382-6.

8. Silverman NS, Greif A. Influenza vaccination during pregnancy. Patients' and physicians' attitudes. J Reprod Med 2001;46:989-94.

9. Schrag SJ, Fiore AE, Gonik B, et al. Vaccination and perinatal infection prevention practices among obstetrician-gynecologists. Obstet Gynecol 2003;101: 704-10.

10. Szilagyi P, Vann J, Bordley C, et al. Interventions aimed at improving immunization rates. Cochrane Database Syst Rev 2002;(4):CD003941.

11. Centers for Disease Control and Prevention. Prevention and control of influenza: recommendations of the Advisory Committee on Immunization Practices (ACIP). MMWR 2003;52(RR-6):1-44. 